\title{
Generation of Atmospheric Pressure Transient Glow Discharge in Microgap Electrode with Nanosecond Pulsed Voltage
}

\author{
Shinji Ibuka Member (Tokyo Institute of Technology, ifuka@ee.titech.ac.jp) \\ Ryo Mikami Member (Tokyo Institute of Technology, mikami@pwr.ee.titech.ac.jp) \\ Fumitaka Furuya Student Member (Tokyo Institute of Technology, furuya@pwr.ee.titech.ac.jp) \\ Kenji Ogura Student Member (Tokyo Institute of Technology, ogura@pwr.ee.titech.ac.jp) \\ Koichi Yasuoka Member (Tokyo Institute of Technology, yasuoka@ee.titech.ac.jp) \\ Shozo Ishii Member (Tokyo Institute of Technology, ishii@ee.titech.ac.jp)
}

Keywords: atmospheric pressure glow discharge, transient glow discharge, microplasma

Atmospheric pressure transient glow discharges in microgap electrodes were generated using high voltage pulse with the nanosecond pulse width ranging from 50 to $300 \mathrm{~ns}$. A short pulse generator using a pulse forming line scheme with a reflected wave absorption circuit was designed and operated.

A stainless steel nozzle cathode and a tungsten carbide sphere anode electrode system was prepared. The inner and the outer diameter of the nozzle were $190 \mu \mathrm{m}$ and $350 \mu \mathrm{m}$ respectively. Applying the high voltage pulse with $50 \mathrm{~ns}$ of pulse width and $1 \mathrm{kHz}$ of the repetition frequency, the stable atmospheric pressure glow discharge (APGD) was successfully obtained with the helium gas flow rate of $150 \mathrm{sccm}$.

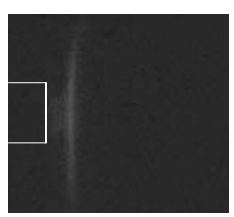

gap length:

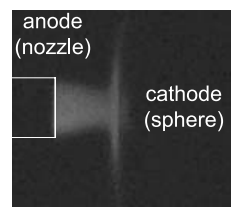

(b) $300 \mu \mathrm{m}$

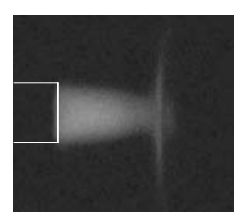

(c) $500 \mu \mathrm{m}$
Fig. 1. Time integrated CCD photographs of the APGD with fast gas flow of helium

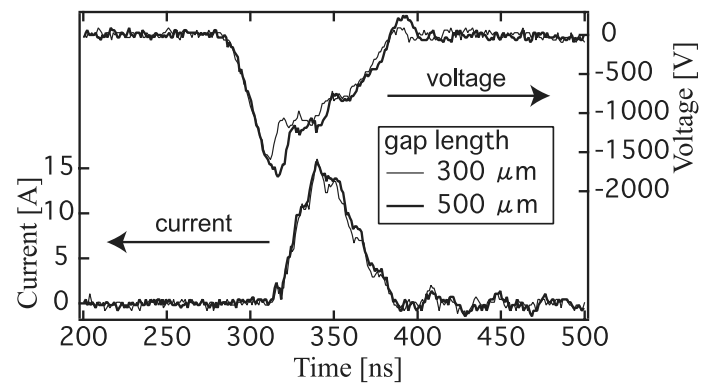

Fig. 2. Voltage and current waveforms of the APGD with fast gas flow of helium

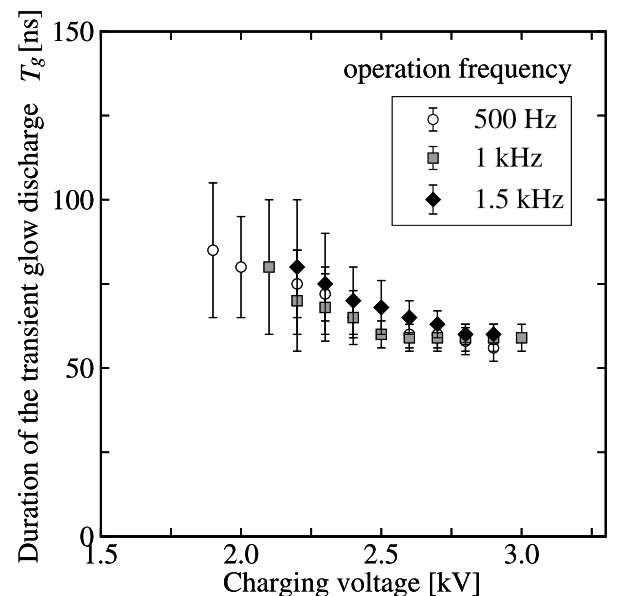

Fig. 3. Duration of the transient glow discharge $T_{g}$ for various charging voltage and driving frequency

Figures 1 and 2 show CCD photographs and the voltage and the current waveforms of the discharge. The high spatial uniformity and the high sustaining voltage are typical features of the generation of the APGD.

The duration of the transient glow discharge $T_{g}$ was estimated and summarized in Fig. 3. The duration $T_{g}$ decreases with increasing the charing voltage because heating in the discharge develops markedly according to the increase of injection energy. The decrease of $T_{g}$, however, becomes saturated to be approximately $60 \mathrm{~ns}$. It suggests that pulse width of shorter than $60 \mathrm{~ns}$ is suitable for generating stable APGD in various voltage range.

In our experimental results, the peak input power is 25 $\mathrm{kW}$, which is an extremely large value compared with 1-10 watts for the direct current APGDs. The utilization of the transient glow discharge extends the application fields of the APGD. 


\section{Generation of Atmospheric Pressure Transient Glow Discharge in Microgap Electrode with Nanosecond Pulsed Voltage}

$\begin{array}{ll}\text { Shinji Ibuka* } & \text { Member } \\ \text { Ryo Mikami* }^{*} & \text { Member } \\ \text { Fumitaka Furuya* }^{*} & \text { Student Member } \\ \text { Kenji Ogura* }^{*} & \text { Student Member } \\ \text { Koichi Yasuoka* }^{*} & \text { Member } \\ \text { Shozo Ishii* } & \text { Member }\end{array}$

Atmospheric pressure transient glow discharges in microgap electrodes were generated using high voltage pulse with the nanosecond pulse width ranging from 50 to 300 ns. A short pulse generator using a pulse forming line scheme with a reflected wave absorption circuit was designed and operated. The transient glow discharges developed to arcs when the pulse width was long enough. Their duration decreased with increasing an ambient gas pressure and extended with shorter electrode separation. Utilizing a nozzle-to-sphere electrode system with fast gas flow of helium, a stable atmospheric pressure transient glow discharge was successfully obtained. The injection energy was controlled by changing the applied voltage and the gap length. The peak input power of $25 \mathrm{~kW}$ was obtained.

Keywords: atmospheric pressure glow discharge, transient glow discharge, microplasma

\section{Introduction}

An atmospheric pressure glow discharge (APGD) is growing concern among researchers in the field of plasma applications, because it has special features appropriate for industrial applications ${ }^{(1)-(3)}$. In general, discharges in high pressure gas tend to shrink into a thin current path forming an arc discharge. A key issue to generate stable APGDs is the suppression of a glow-to-arc transition, for which various techniques have been proposed and tested so far, such as forced cooling of electrodes, utilization of high frequency voltage, dielectric barrier discharges, and external electron sources ${ }^{(4)-(7)}$. Applying those methods, the APGD has been generated with air, nitrogen, and noble gases. The stabilization mechanism of APGD has been also investigated with numerical analysis ${ }^{(8)(9)}$. F. Massines et al reported the difference in the mechanism between helium and nitrogen discharges ${ }^{(9)}$. Helium, which has a metastable state of high energy level, is advantageous to the APGDs. Large volume APGDs with high spatial uniformity is required for the plasma applications. It is, however, difficult to obtain the large volume APGD because spatial instabilities grow and uniformity will be distorted.

On the other hand, generation of plasmas with a small scale has also attracted significant interests ${ }^{(10)-(14)}$. The plasmas produced in the microgap electrode are known as microplasmas. The microplasmas are in the size of

\footnotetext{
* Tokyo Institute of Tecnology

2-12-1, O-okayama, Meguro-ku, Tokyo 152-8552
}

10-1000 $\mu \mathrm{m}$ and have a very large specific surface area. This enhances the energy losses to the wall or ambient gases, consequently the microplasmas are usually in a non-equilibrium state. The non-equiliblium plasmas with the small scale have advantages for applications of plasma chemistry and plasma processing, such as maskless etching of silcon, surface modifications of polymeric film substrates, plasma sterilization and so on ${ }^{(10)(15)-(17)}$. Furthermore, the small size of the microplasma obstructs the growth of spatial instability. The microplasma is one of a promising candidate to generate the stable APGD. K. Schoenbach et al proposed a micro hollow cathode configuration to generate the high density APGD ${ }^{(11)}$. T. Yokoyama et al reported the utilization of a miniature gas flow to obtain direct current APGDs with helium and argon ${ }^{(12)}$. Their method is advantageous for applications such as thin-film deposition and plasma fine processing because the microplasma is generated without a confining wall. Although a simple apparatus of the direct current driving method is preferable for the industrial applications, the difficulty in the control of the injection energy to the microplasma causes the limitation of the application area.

In this study, we propose to utilize a transient glow discharge with short pulsed voltage of several hundreds nanosecond in pulse width to generate the high power APGD microplasmas. The transient glow discharge is one of the most well-known techniques to obtain the high pressure glow plasma ${ }^{(18)}$. The pulsed voltage is made zero before the glow-to-arc transition. It is possible to generate the high power density glow plasma. 
However, there have been few reports concerning the discharge characteristics in the phased of the glow-toarc transition in the microgap of less than $1 \mathrm{~mm}^{(14)}$. In this paper, we report the preliminary experiments for the transient glow micro discharge and the feasibility of obtaining the high power density APGD.

\section{Experimental Setup}

Figure 1 shows a schematic of the experimental apparatus for the discharges under various gas pressures. The electrodes were installed in a vacuum chamber in order to examine the effects of the working gas pressure on the discharges. The chamber was evacuated to $10^{-2}$ Torr with a vacuum pump before filling the working gas. The atmospheric pressure experiments with gas flow were conducted in open air without the chamber. The gas flow rate was regulated with a mass flow controller.

The miniature electrode system was assembled in conjunction of metal electrodes, which include a sphere and a needle of tungsten carbide and nozzle of stainless steel as shown in Fig. 2. The diameter of the sphere was 2 $\mathrm{mm}$ and the radius of curvature of the needle was $50 \mu \mathrm{m}$. The inner and the outer diameter of the nozzle electrode were $190 \mu \mathrm{m}$ and $350 \mu \mathrm{m}$ respectively. Helium or argon

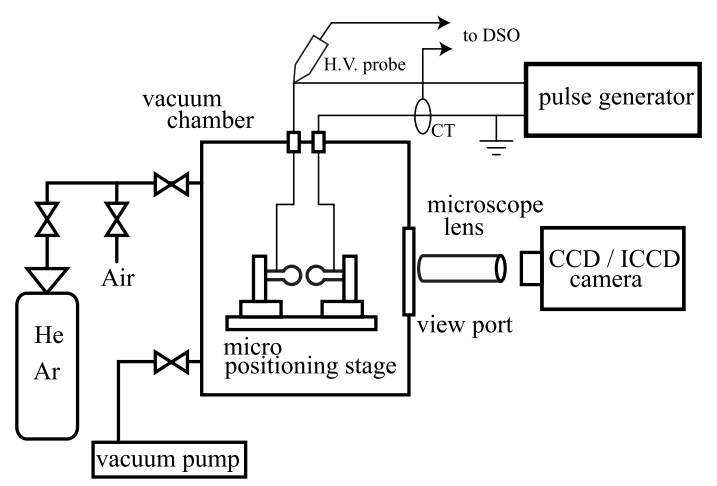

Fig. 1. The schematic of the experimental apparatus

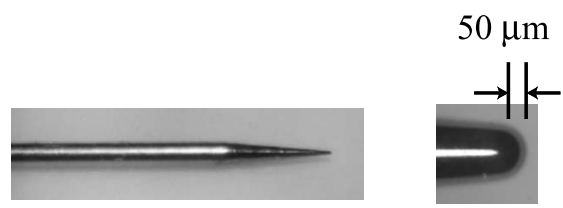

(a) needle

enlarged view of the tip

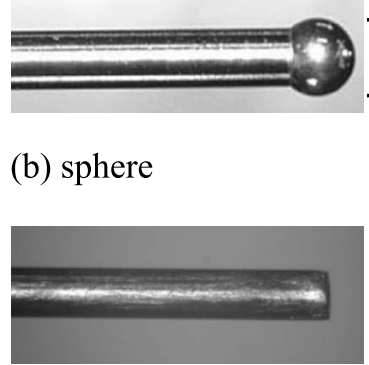

$2 \mathrm{~mm}$

(c) nozzle

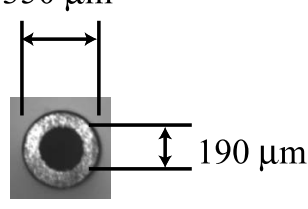

enlarged cross sectional view

Fig. 2. The shapes of the electrodes

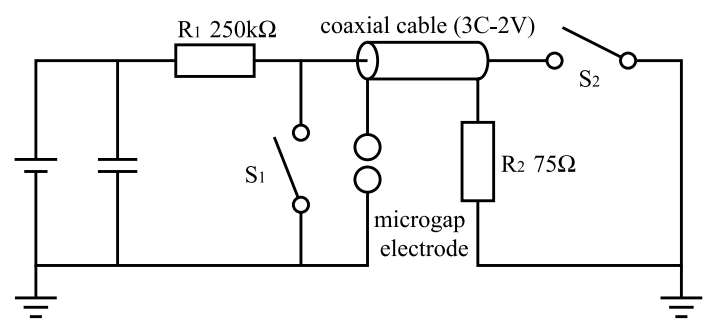

Fig. 3. The circuit diagram of the pulse generator

gas flowed out of the nozzle in air. All the electrodes were placed on the micro positioning stages to control the electrode separation in range of micron oder. The applied voltage to the electrodes was monitored by a high voltage probe (PMK GmbH PHVS622: $400 \mathrm{MHz}$ BW). A current monitor (Pearson 411: $20 \mathrm{MHz}$ BW) was used to measure the current waveform. The voltage and the current waveform were recorded by a digital storage oscilloscope (LeCroy WavePro 960: $2 \mathrm{GHz} \mathrm{BW}$ ). The discharge was monitored by a CCD camera with a microscope lens because the size of microplasmas was less than $1 \mathrm{~mm}$ and observing them by the naked eye was difficult. An image intensified CCD (ICCD) camera was also used to observe temporal and spatial behavior of the discharges.

Figure 3 is a circuit diagram of the pulse generator to power the discharges ${ }^{(14)}$. A pulse forming line scheme with a $3 \mathrm{C}-2 \mathrm{~V}$ coaxial cable enables us to generate a short pulse width of 50-300 ns. In general, the load impedance of the discharge plasma decreases to $10-20 \Omega$. It is difficult to obtain impedance matching completely between the generator and the load. Therefore, we proposed to utilize a reflected wave absorption circuit at the open end of the cable. When the switches, $\mathrm{S}_{1}$ and $\mathrm{S}_{2}$, are closed simultaneously, a pulsed voltage appears between the electrodes and the discharge develops. The reflected wave is originated due to the mismatching of the load impedance and propagates along the cable backward to the other end. At the end of the cable, the reflected wave is fully absorbed by the resistance $R_{2}$ because the resistance of $R_{2}$ is set to be $75 \Omega$ which is the same value of the characteristic impedance of the $3 \mathrm{C}-2 \mathrm{~V}$ coaxial cable. A single square pulsed voltage with the pulse width of less than $300 \mathrm{~ns}$ is, consequently, applied to the electrode. The rise time of the pulse is $30 \mathrm{~ns}$. To realize highly repetitive operation, stacked power semiconductor devices were used as the switches. The energy transfer efficiency of the pulse generator was not high because the half of the stored energy in the cable is consumed in the resistor $R_{2}$. To improve the energy transfer efficiency, utilizing a Blumlein line configuration and controlling the switching timing of $\mathrm{S}_{2}$ is effective. However, that is beyond the scope of this paper. It should be investigated in the future work.

\section{Results and Discussions}

3.1 Pressure Dependence of the Transient Glow Discharge Figure 4 shows the voltage, current, power and injected energy waveforms in the 


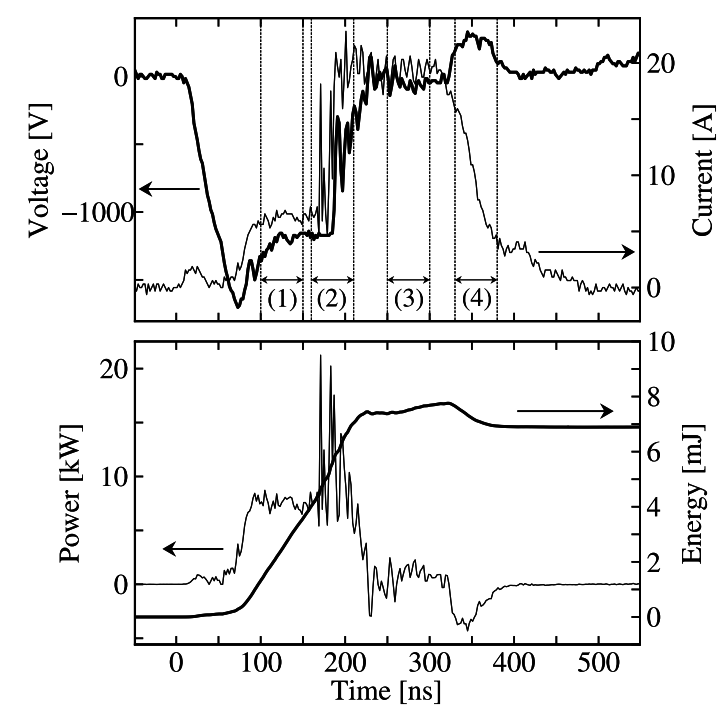

Fig. 4. Voltage, current, power and injected energy waveforms of the air discharge in the pressure of 100 Torr; Numbers in the figure indicate the gate timing of figure 5 (voltage and energy: thick line, current and power: thin line)

discharge with the needle-to-needle electrode system in 100 Torr air. The pulse width of the pulse generator was set to be $300 \mathrm{~ns}$ using the cable of $60 \mathrm{~m}$ in length. The cable was charged to $2.9 \mathrm{kV}$ and the initial stored energy was $16.8 \mathrm{~mJ}$. The unstable filamentary discharge was observed in the time integrated CCD photographs. However, the formation of the transient glow discharge was recognized in the temporal change in the voltage and the current. In general, the glow and the arc discharge are defined by their discharge maintenance mechanism. The glow discharge is maintained generally by the secondary electron emission and the impact ionization. In other hand, the thermal ionization and the thermionic emission are dominant in the arc discharge. However, the recent novel glow-like discharges are not fully studied from the viewpoint of the discharge maintenance mech$\operatorname{anism}^{(19)}$. In this paper, we defined the glow discharge as the spatially uniform discharge with high sustaining voltage. As shown in Fig. 4, under the half of the stored energy was transfered to the plasma. Most of the rest energy was consumed in the resistance $R_{2}$.

Figure 5 shows time resolved photographs of the discharge taken using the ICCD camera with the exposure time of 50 ns. Although each photograph was taken in the different shots of the repetitive discharges having high reproducibility, the set of photographs reproduces the sequential change of the discharge. The gate timing of the ICCD camera is indicated in Fig. 4. In the first frame (100-150 ns), a spatially uniform transient glow discharge extending to the surface of the cathode with high sustaining voltage is obtained, where a sustaining voltage is high and is $1200 \mathrm{~V}$. Glow-to-arc transition is observed in the second frame (160-210 ns). A bright spot was formed at the tip of the cathode. In the third frame (250-300 ns), the discharge is shrunk to a thin current path and the applied voltage decreases to zero.

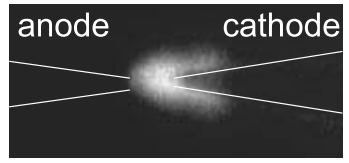

(a) $100 \sim 150 \mathrm{~ns}$

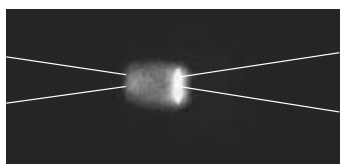

(c) $250 \sim 300 \mathrm{~ns}$

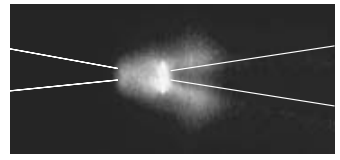

(b) $160 \sim 210 \mathrm{~ns}$

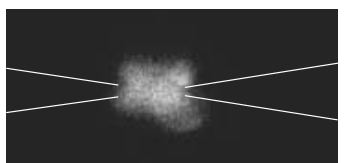

(d) $330 \sim 380 \mathrm{~ns}$
Fig. 5. ICCD photographs of the air discharge with exposure time of $50 \mathrm{~ns}$ in the pressure of 100 Torr

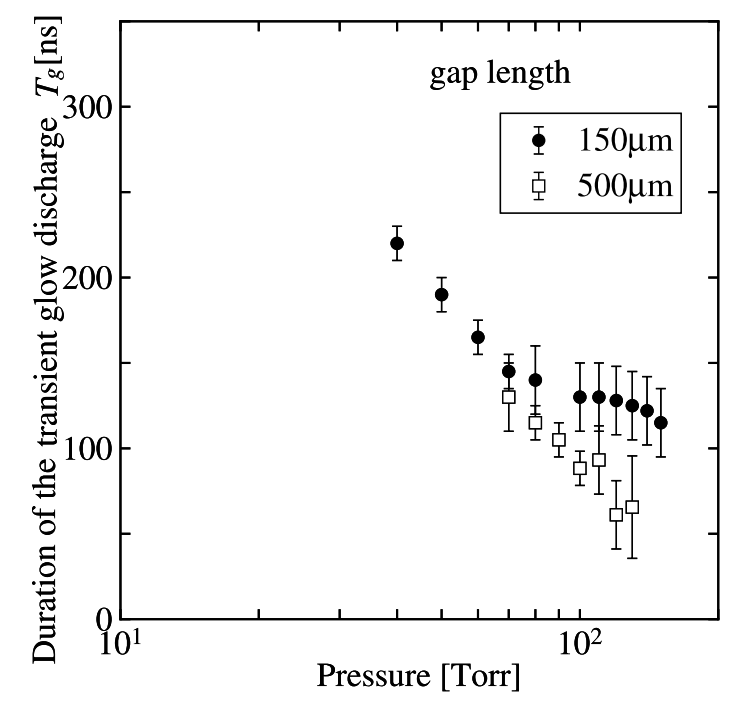

Fig. 6. Duration of the transient glow discharge $T_{g}$

In the last frame (330-380 ns), the discharge shows diffusive profile, which is a typical behavior of the afterglow.

Here, we define the duration of the transient glow discharge $T_{g}$ as the time between the breakdown and the beginning of the glow-to-arc transition, at which the voltage starts to decrease to zero. Figure 6 shows the dependence of the $T_{g}$ on the ambient air pressure. $T_{g}$ decreased as the pressure is increased. The gap length also affected the duration. According to the results of Fig. 6, the short gap length has advantage of obtaining the transient glow discharge.

3.2 Influence of Ambient Gas Species The discharge characteristics with helium or argon were similar to those with air. The occurrence probability of the glow-to-arc transition was lowered as compared with that of the air discharges. Figure 7 shows the voltage, current and injected energy waveforms for helium and argon discharge at the pressure of 50 Torr with the sphere-to-sphere electrode system. A marked difference in helium and argon discharge is the rise time of the discharge current. In the case of helium, the discharge current increased slowly and sustaining voltages were higher than those for argon discharge. These differences are based on the higher ionization voltage of helium. In dc or high duty ratio pulsed discharges, the cumulative ionization of helium is dominant and the discharge is 


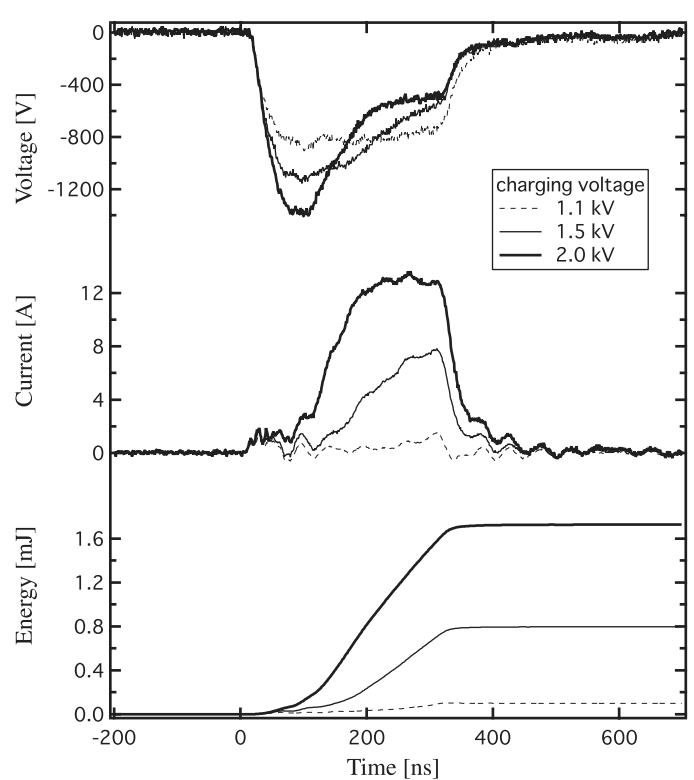

(a) He (pressure: 50Torr, Gap length: $150 \mu \mathrm{m}, 500 \mathrm{~Hz}$ )

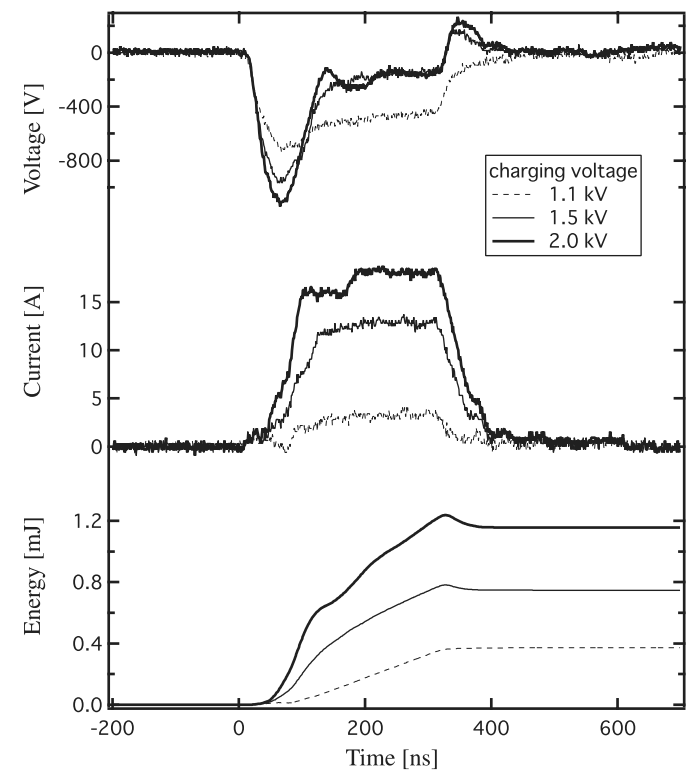

(b) Ar (pressure: 50Torr, Gap length: $150 \mu \mathrm{m}, 500 \mathrm{~Hz}$ )

Fig. 7. Voltage, current and injected energy waveforms of helium and argon discharge

sustained at a lower voltage than that in our experiment.

3.3 Generation of APGD We examined the atmospheric pressure transient glow discharge with the gas flow of air, helium, or argon using the short width pulsed voltage. No stable glow discharge without the glow-to-arc transition was obtained for air and argon gas flow. The rapid voltage drop and shrinking of the discharge with the bright spot around the cathode tip as shown in Fig. 5(c) indicates the occurrence of the glowto-arc transition. Frequent energy exchange of particles by collision accelerates the glow-to-arc transition. Local heating of the electrode surface owing to high repetition rate operation also hampered the development of glow discharges. In the previous report of the direct current APGD with the miniature electrodes, fast gas

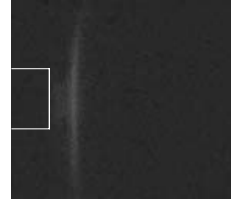

gap length:

(a) $100 \mu \mathrm{m}$

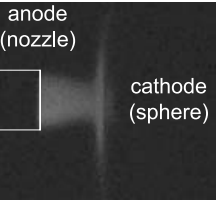

(b) $300 \mu \mathrm{m}$

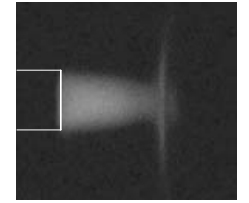

(c) $500 \mu \mathrm{m}$
Fig. 8. Time integrated CCD photographs of the APGD with fast gas flow of helium

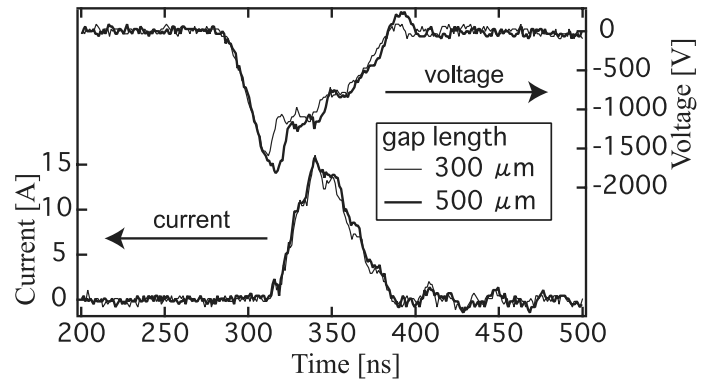

Fig. 9. Voltage and current waveforms of the APGD with fast gas flow of helium

flow enhanced the discharge stability drastically ${ }^{(12)}$. In this study, we used a helium gas flow with the nozzle-tosphere electrode system. Figure 8 shows the CCD photographs of the helium gas flow discharge, where the gap separation was changed from $100 \mu \mathrm{m}$ to $500 \mu \mathrm{m}$. The gas flow rate was set to be $150 \mathrm{sccm}$. The discharges were powered by the high voltage pulses with the pulse width of $50 \mathrm{~ns}$ and the repetition rate of $1 \mathrm{kHz}$. In all photographs, a weakly emitting region covers the surface of the sphere cathode. The luminous column bridges the electrodes for the 300 and $500 \mu \mathrm{m}$ gap discharges. This corresponds to a positive column, because the similar structure is obtained in the direct current APGD ${ }^{(12)}$. Figure 9 shows the voltage and the current waveforms for the gap length of 300 and $500 \mu \mathrm{m}$. The high spatial uniformity and the high sustaining voltage are typical features of the generation of APGD. The similar result was obtained in the discharge with the gap length of up to $600 \mu \mathrm{m}$. For longer gap length over $700 \mu \mathrm{m}$, the discharge became unstable and the glow-to-arc transition occurred.

Figure 10 shows the CCD photograph of the discharge and the voltage and current waveforms with the long pulse width voltage using the $30 \mathrm{~m}$ cable as the PFL. The glow-to-arc transition was observed, following a transient glow discharge. A bright spot was formed on the cathode surface and the discharge shape became similar to the arc discharge. However, the voltage and the current waveforms indicate the formation of the transient glow discharge before the glow-to-arc transition. Figure 11 shows the duration of the transient glow discharge $T_{g}$ dependence on charging voltages and repetition frequencies. The absence of the plot in the low voltage region indicates that the glow-to-arc transition does not occur at those parameter regions. The duration $T_{g}$ decreases with increasing the charing voltage. This is explained 


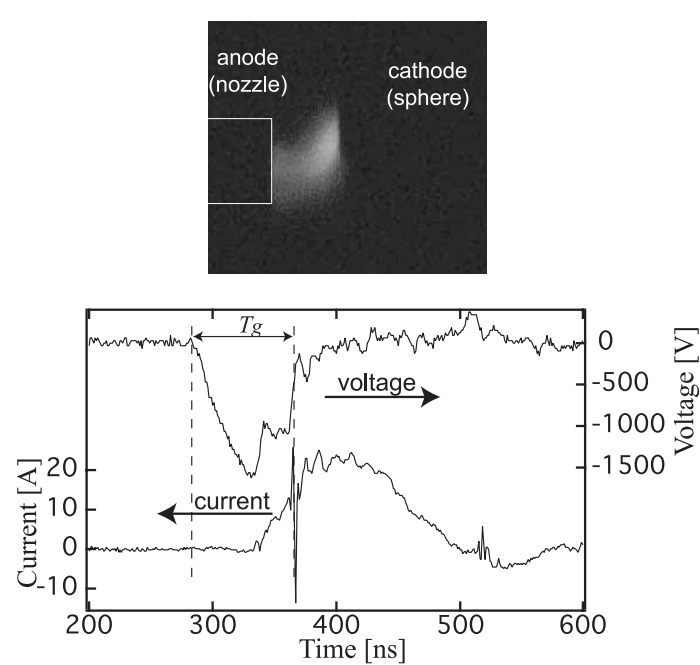

Fig. 10. Time integrated CCD photograph of the discharge and voltage and current waveforms of the discharge driven by long pulse

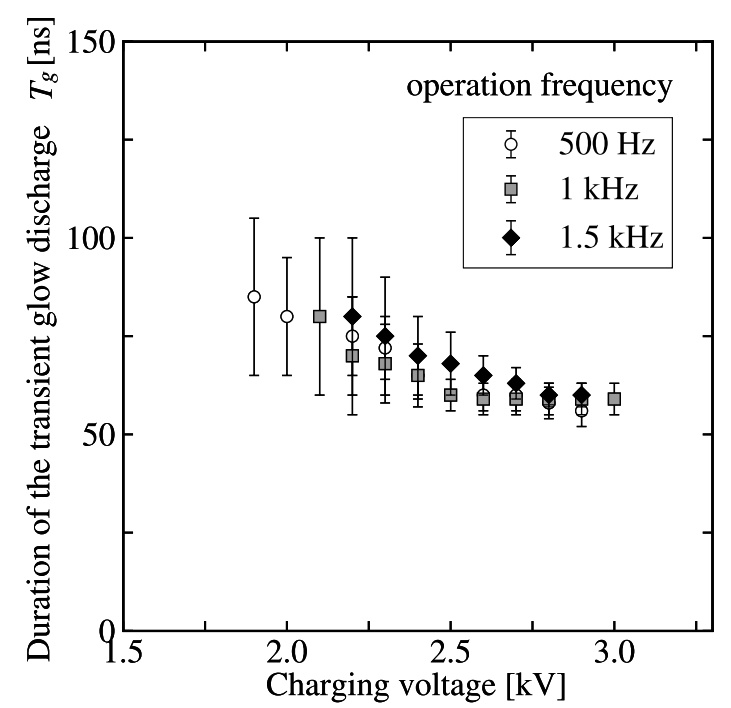

Fig. 11. Duration of the transient glow discharge $T_{g}$ for various charging voltage and driving frequency

by that heating in the discharge develops markedly according to the increase of injection energy. The decrease of $T_{g}$, however, become saturated to be approximately $60 \mathrm{~ns}$. It suggests that the shorter pulse width than $60 \mathrm{~ns}$ is suitable for generating stable APGD in various voltage range. In other literatures, it was discussed that the metastable helium atom contributes to improving the stability of the glow discharge ${ }^{(9)}$. In this study, however, the repetition frequency does not affect $T_{g}$ because the residence time of the gas in the electrode gap is estimated as $1.2-6.0 \mu \mathrm{s}$ for the electrode separation of $100-500 \mu \mathrm{m}$.

There is a possibility to control the injection energy to the APGDs. We calculated the injection energy of a single pulse by integrating the products of the voltage and the current. Figure 12 shows the gap length dependence

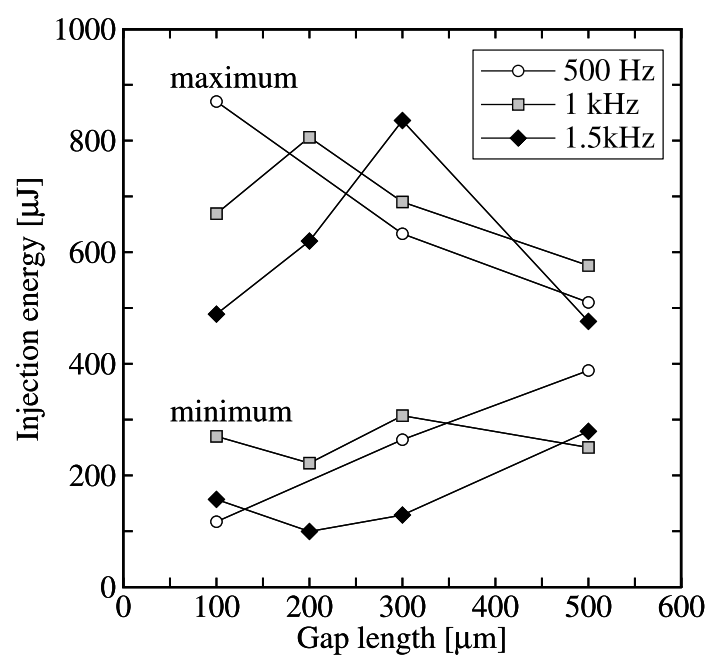

Fig. 12. The maximum and the minimum injection energy of the stable glow discharge

of the maximum and the minimum injection energy in the stable glow discharges, between which the injection energy can be controlled by changing the applied voltage. The controllable region extends with shortening the gap length, except for $1.5 \mathrm{kHz}$, where the resonance of circuit will affect the discharge characteristics. The reason, however, is not fully discussed yet. We demonstrated that the short gap length is effective to generate stable APGDs. The peak input power to the discharge is also an important parameter for plasma applications. In our experimental results, the peak input power is 25 $\mathrm{kW}$, which is an extremely large value compared with 1-10 watts for the direct current APGDs ${ }^{(12)(13)}$. The utilization of the transient glow discharge extends the application fields of the APGD.

\section{Summary and Conclusions}

Utilizing the PFL type short pulse generator with the reflected wave absorption circuit, the transient glow discharge in the microgap electrode was experimentally investigated. The duration of the transient glow discharge decreased as the increase of the ambient gas pressure and extended with shorter electrode separation. Introducing fast helium gas flow with the driving pulse width of 50 ns, the stable APGD was successfully obtained with the gap length of less than $600 \mu \mathrm{m}$. The injection energy of the APGD could be adjusted in wide range by varying the driving voltage. The variable range of the injection energy was enlarged with shorter electrode gap length. The experimental results illustrated the effectiveness of applying the transient glow discharge to the microgap electrode discharge.

\section{Acknowledgment}

This work was supported by Grant-in-Aid for Scientific Research from Ministry of Education, Culture, Sports, Science and Technology.

(Manuscript received June 30, 2006, revised Feb. 13, 2007) 


\section{References}

(1) M. Kogoma, T. Nozaki, K. Okazaki, and Y. Sawada: "Applications of Atmospheric Pressure Glow Plasma," J. Plasma Fusion Res., Vol.79, No.10, pp.1009-1028 (2003) (in Japanese)

(2) V.A. Titov, V.V. Rybkin, T.G. Shikova, T.A. Ageeva, O.A. Golubchikov, and H.S. Choi: "Study on the application possibilities of an atmospheric pressure glow discharge with liquid electrolyte cathode for the modification of polymer materials," Surface \& Coatings Technology, Vol.199, pp.231-236 (2005)

(3) M. Vleugels, G. Shama, X.T. Deng, E. Greenacre, T. Brocklehurst, and M.G. Kong: "Atmospheric Plasma Inactivation of Biofilm-Forming Bacteria for Food Safety Control," IEEE Trans. Plasma Sci., Vol.33, No.2, pp.824-828 (2005)

(4) S. Kanazawa, M. Kogoma, T. Moriwaki, and S. Okazaki: "Stable glow plasma at atmospheric pressure," J. Phys. D: Appl. Phys., Vol.21, pp.838-840 (1988)

( 5 ) K. Takaki, T. Fujiwara, and F. Tochikubo: "Production of Atmospheric-Pressure Glow Discharge," J. Plasma Fusion Res., Vol.79, No.10, pp.1002-1008 (2003) (in Japanese)

(6) K. Takaki, M. Hosokawa, T. Sasaki, S. Mukaigawa, and T. Fujiwara: "Production of atmospheric-pressure glow discharge in nitrogen using needle-array electrode," Appl. Phys. Lett., Vol.86 (2005)

( 7 ) J.J. Shi and M.G. Kong: "Large-volume and low-frequency atmospheric glow discharges without dielectric barrier," Appl. Phys. Lett., Vol.86 (2005)

(8) F. Tochikubo, T. Chiba, and T. Watanabe: "Structure of LowFrequency Helium Glow Discharge at Atmospheric Pressure between Parallel Plate Dielectric Electrodes," Jpn. J. Appl. Phys., Vol.38, No.9A, pp.5244-5250 (1999)

(9) F. Massines, P. Segur, N. Gherardi, C. Khamphan, and A. Ricard: "Physics and chemistry in a glow dielectric barrier discharge at atmospheric pressure: diagnostics and modelling," Surface \& Coatings Technology, Vol.174-175, pp.8-14 (2003)

(10) K.H. Becker, K.H. Schoenbach, and J.G. Eden: "Microplasmas and applications," J. Phys. D: Appl. Phys., Vol.39, pp.R55-R70 (2006)

(11) K.H. Schoenbach, A.E. Habachi, W. Shi, and M. Ciocca: "High-pressure hollow cathode discharges," Plasma Sources Sci. Technol., Vol.6, pp.468-477 (1997)

(12) T. Yokoyama, S. Hamada, S. Ibuka, K. Yasuoka, and S. Ishii: "Atmospheric dc discharges with miniature gas flow as microplasma generation method," J. Phys. D: Appl. Phys., Vol.38, pp.1684-1689 (2005)

(13) D. Staak, B. Farouk, A. Gutsol, and A. Fridman: "Characterization of a dc atmospheric pressure normal glow discharge," Plasma Sources Sci. Technol., Vol.14, pp.700-711 (2005)

(14) R. Mikami, F. Furuya, S. Ibuka, K. Koichi, and S. Ishii: "Highfrequency pulse generator with a distributed constant line and its application to atmospheric microplasma generation," The Papers of Technical Meeting on Plasma Science and Technology, IEE Japan, pp.47-52 (2005) (in Japanese)

(15) T. Ichiki, R. Taura, and Y. Horiike: "Localized and ultrahighrate etching of silicon wafers using atmospheric-pressure microplasma jets," J. Appl. Phys., Vol.95, pp.35-39 (2004)

(16) E. Stoffels, A.J. Flikweert, W.W. Stoffels, and G.M.W. Kroesen: "Plasma needle: a non-destructive atmospheric plasma source for fine surface treatment of (bio)materials," Plasma Sources Sci. Technol., Vol.11, pp.383-388 (2002)

(17) C. Schrader, P. Sichler, L. Baars-Hibbe, N. Lucas, A. Schenk, S. Draeger, K.H. Gericke, and S. Buttgenbach: "Microstructured electrode arrays: Plasma based sterilization and coating over a wide pressure range," Surface \& Coatings Technol., Vol.200, pp.655-659 (2005)

(18) J. Sekikawa and T. Fujiwara: "Cross-Sectional Area and Current Density of Transient Glow Discharge after Static Breakdown," T. IEE Japan, 118-A, 9, pp.986-990 (1998) (in Japanese)

(19) Investigating R\&D committee on fundamental technologies of arc and glow discharge: Fundamental technologies of arc and glow discharges -investigations on recent technologies over whole fields of these discharges-, IEEJ Technical Report, No.1066 (2006) (in Japanese)

Shinji Ibuka

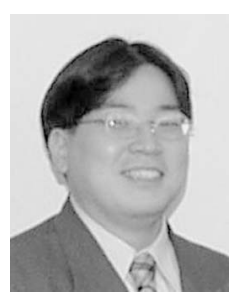

(Member) He was born on January 30, 1969 He received the B.E. and M.E. degrees from Tokyo Institute of Technology in 1992 and 1994, respectively. He is a research associate in the Tokyo institute of technology. His research interests have included plasma applications, pulsed power technology and highvoltage semiconductor switches.

Ryo Mikami

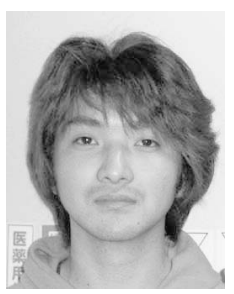

(Member) He was born on July 5, 1981. He received the B.E. degree from Shibaura Institute of Technology in 2004. He received the M.E. degree from Tokyo Institute of Technology in 2006. He is currently employed by Canon Inc..

Fumitaka Furuya (Student Member) He was born on April

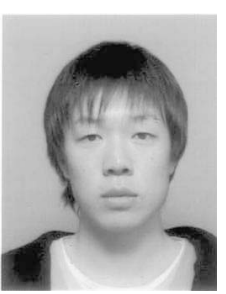
5, 1982. He received the B.E. degree from Tokyo Institute of Technology in 2005. He is currently studying as a master course student at Tokyo Institute of technology.

Kenji Ogura

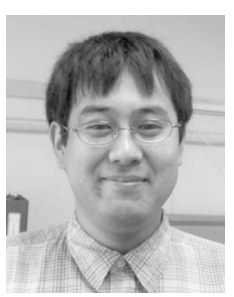

Student Member) He was born on June 4, 1982. He is currently studying as an under graduate student at Tokyo Institute of technology.

Koichi Yasuoka (Member) He was born on September 22,

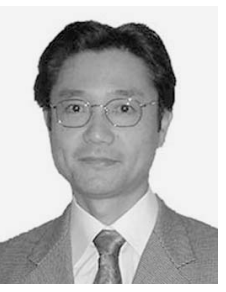
1955. He receive the M.E and D.E. degrees from Tokyo Institute of Technology, Japan, in 1980 and 1983, respectively. He is an associate professor in the Department of Electrical and Electronic Engineering, Tokyo Institute of Technology. His current research interests include generation and utilization of atmospheric plasmas with dc and microwave technology

Shozo Ishii (Member) He was born on December 16, 1945.

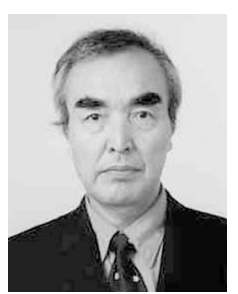

He received the M.E. and D.E. degrees from Tokyo Institute of Technology, Japan, in 1970 and 1973, respectively. He is a professor in the Department of Electrical and Electronic Engineering, Tokyo Institute of Technology. His research field includes plasma physics and high voltage engineering. 